\title{
Tolerance-based Structural Design of Tubular-Structure Loading Equipments
}

\author{
Jiping Lu, Shuiyuan Tang*, Jianhua Zuo, Hongli Fan \\ School of Mechanical Engineering, Beijing Institute of Technology, \\ Beijing 100081, China \\ Zhonghua Jiang \\ Institute of Science \& Technology, China North Industries Group Corporation \\ Beijing 100089, China \\ Shahid I Butt \\ National University of Sciences \& Technology, Rawalpindi, Pakistan \\ Received: 25-02-2011 \\ Accepted: 18-04-2011
}

\begin{abstract}
Mechanical loading equipments are wildly used in transportation system. Positioning precision is one of the basic functions of machine tools, and structure design is the key to ensuring accuracy. According to the assembly process of tubular-structure, the paper analyzes motion modes to achieve tubular-structure loading. In terms of assembly tolerance of tubular-structure, a tolerance model to guide structure design of loading equipment is formulated. Based on tolerance analysis of each motion mode, the maximum available size of the tubular-structure is calculated under different linear rolling guide, and the minimum available size of the interior rail in the cover box is worked out under different ball screws, trapezoidal screw threads, worm and worm gears. To meet the requirement of tolerance in tubular-structure assembly, mechanisms for all motions are defined. The design of loading equipment is tested and assessed by experiments, and the result shows the design is highly qualified for its assembly.
\end{abstract}

Key words: tolerance allocation, structure design, tubular-structure, loading equipment.

\section{Introduction}

Tolerance plays a vital role in product design and manufacturing. Proper allocation of tolerance among the components of a mechanical assembly is a promising approach to reduce manufacturing cost in a large scale production. Considerable research works have been published on optimal tolerance synthesis for simple and complex assemblies. For instance, Peters (1970) made an extensive study of different possibilities of tolerances distribution among parts of an assembly in order to obtain minimum cost ${ }^{1}$. Speckhart (1972) presented an analytical method to carry out the optimum set of dimensional tolerances for a mechanical device minimizing the manufacturing cost and meeting the imposed constraint conditions $^{2}$. Tolerance assignment on dimensions of manufactured parts has profound influence on manufacturing cost. In general, tighter tolerances result in higher manufacturing costs ${ }^{3}$. Inappropriate tolerance specification may also result in poor product performance.

The precision of assembly equipments affects considerably assembly quality and product's performance. Assembly processes and equipments are usually defined in accordance with product tolerance specification and tolerance allocation. Publications focus primarily on product tolerance design and its allocation. However, it is seldom reported how to design assembly equipment when assembly tolerance is given. Kim and Knott (1987) conducted a study of positional tolerance of peg-and-hole assembly ${ }^{4}$. Doydum and Perreira (1991) analyzed assemblies with irregular cross-sections based on Monte Carlo simulation ${ }^{5}$. Cho and Tu (2002) developed a sys-

\footnotetext{
*Corresponding author: shuiyuantang@bit.edu.cn
} 
tematic method of assigning circularity tolerance based on functional requirements and process capability in manufacturing ${ }^{6-9}$.

Machine tolerances can lead to position errors, uncertain behavior and other undesirable effects ${ }^{10-12}$. How these tolerances affect the output of a machine becomes an important problem ${ }^{13-15}$. This paper presents an approach to modeling and analyzing tolerance for designing assembly equipments. According to the requirements of tubular-structure assembly tolerance specification, loading equipment is divided into components which tolerance is synthesized into equipment precision. Based on movement analysis, we put forward some feasible design solutions. Finally the loading equipment for tubular-structure is designed, and the experiment data shows the equipment is satisfied with the tubular-structure assembly tolerance.

\section{Tolerance-based design principles of} tubular-structure loading equipments

\subsection{Tubular-structure loading process}

The tubular-structure loading equipment is an assembly tools, which is used to push into and pull out tubular-structure of the box. It is important and necessary to analyze equipment's functions, behaviors and structures. The function of loading equipment is a statement of core information characterizing the intention of designer. The intention describes the intended task, activity or work which the equipment will be able to perform after it has been manufactured. The behavior refers to operational process of the equipment under its working environment.

The structure refers to a physical system, a sub-assembly, a component or a number of components, which are integrated into a mechanical product. The structure is the support of the behavior, while the function is realized by the behavior, which is restricted by the assembly tolerance.

The tubular-structure loading process includes three steps. The first step is to lay its cover box on the loading

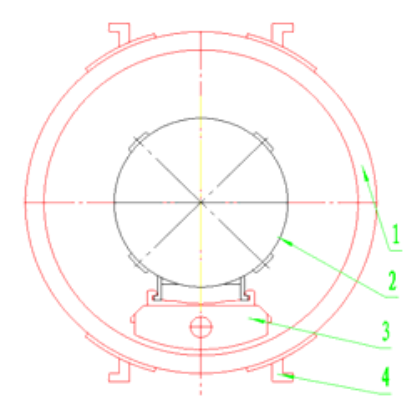

1-cover box, 2-tubular-structure, 3-interior rail, 4-pothook Figure1 Position relationship between tubular-structure and cover box equipment, and adjust box position to satisfy the assembly requirements. The second step is to load a tubular-structure and deposit it on the equipment. The third step is to push the tubular-structure into the loading box. Only the pothooks of the tubular-structure are consistent with the interior straight-line guide rails, could a tubular-structure be pushed into the cover box. When loaded into the box, a tubular-structure is positioned as shown in Figure 1.

\subsection{Design tolerance and requirements of the loading equipment}

In order to push tubular-structure into the cover box without destroying the interior rail, the cover box must be adjusted in several directions. The length of tubular-structure is more than 1 meter. However, the assembly tolerance of pothook and rail is $\pm 2 \mathrm{~mm}$. The movement precision of cover box in different directions determines whether a tubular-structure can be pushed into the box matching specific assembly tolerance. According to the tolerance theory, structural design of tubular-structure loading equipment should follow the criteria that the maximal outer available size of the tubular-structure is smaller than the minimum inner effective size of the cover box, under constraint of assembly tolerance of tubular-structure. In order to design the move modes and mechanical structure of the loading equipment, it is necessary to analyze the tolerance model of the equipment.

According to the functional requirements, the loading equipment must be moved in six directions, including linear movement in axis $\mathrm{X}, \mathrm{Y}$ and $\mathrm{Z}$, revolving movement around axis $\mathrm{X}, \mathrm{Y}$ and $\mathrm{Z}$. $\mathrm{X}$ axis is the axial direction of tubular-structure, its linear movement is driven by electric power or manpower. While linear movements in axis $\mathrm{Y}$ and $\mathrm{Z}$, and revolving movements around axis $\mathrm{X}$, $\mathrm{Y}$ and $\mathrm{Z}$ are driven by manpower.

\section{3 symbols}

$(\psi, \theta, \varphi)$ Eulerian angles of a local coordinate system

$A^{s i}$ transforming matrix from the world coordinate system to a local coordinate system

$T^{m} \quad$ position transforming matrix of the tubular-structure

$\delta_{x}^{m} \quad$ tolerance value of tubular-structure motion in axial $\mathrm{X}$ direction

$\delta_{y}^{m} \quad$ tolerance value of tubular-structure motion in axial Y direction

$\delta_{z}^{m} \quad$ tolerance value of tubular-structure motion in axial $\mathrm{Z}$ direction

$\delta_{x}^{b} \quad$ tolerance value of the box motion in axial $\mathrm{X}$ direction

$\delta_{y}^{b} \quad$ tolerance value of the box motion in axial Y direction 
$\delta_{z}^{b} \quad$ tolerance value of the box motion in axial $\mathrm{Z}$ direction

$\Delta \alpha^{m} \quad$ rolling angle around $\mathrm{X}$ axis when tubular-structure moves

$\Delta \beta^{m} \quad$ rolling angle around $\mathrm{Y}$ axis when tubular-structure moves

$\Delta \gamma^{m} \quad$ rolling angle around $\mathrm{Z}$ axis when tubular-structure moves

$\Delta \alpha^{b} \quad$ tolerance value of the box rotation around $\mathrm{X}$ axis

\section{Tolerance modeling for tubular-structure loading equipments}

According to the requirements of tubular-structure assembly process, the loading equipment should have the following functions:

(i) Support the tubular-structure and the cover box,

(ii) Move the tubular-structure along its axis,
$W_{\max }^{m} \quad$ the maximum width of tubular-structure

$H_{\max }^{m} \quad$ the maximum height of tubular-structure

$W_{\min }^{b} \quad$ the minimum width of the interior rail

$H_{\min }^{b} \quad$ the minimum height of the interior rail

$\mathrm{X}^{\mathrm{i}} \mathrm{Y}^{\mathrm{i}} \mathrm{Z}^{\mathrm{i}}$ the world coordinate system

$\mathrm{X}^{\mathrm{m}} \mathrm{Y}^{\mathrm{m}} \mathrm{Z}^{\mathrm{m}}$ the local coordinate system for tubular-structure

$\mathrm{X}_{1}^{\mathrm{b}} \mathrm{Y}^{\mathrm{b}}{ }_{1} \mathrm{Z}^{\mathrm{b}}{ }_{1}$ the local coordinate system for the box $\mathrm{X}_{2}^{\mathrm{b}} \mathrm{Y}_{2}^{\mathrm{b}} \mathrm{Z}_{2} \mathrm{~b}$ the local coordinate system for the box

(iii) Move the cover box along its axis,

(iv) Adjust the attitude of the cover box, and

(v) Send out an alarm when danger happens.

In order to achieve the above functions the equipment should include a tubular-structure support frame, a box support frame, two tubular-structure driven blocks, and two box adjusting machines. The principle structural configuration of the equipment is shown in Figure 2.

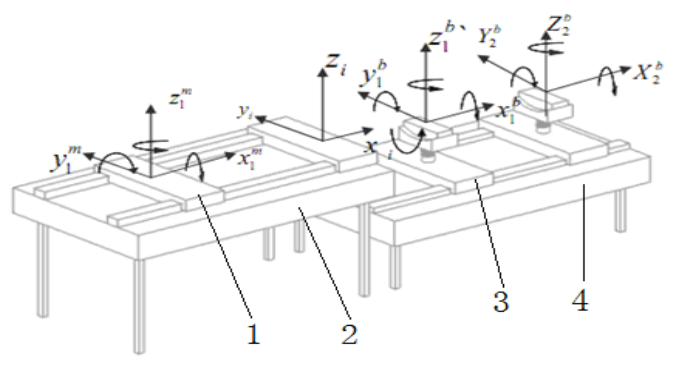

1-tubular-structure seat, 2-support platform for tubular-structure, 3-box seat, 4-support platform for cover box

Figure 2 Principle structural configuration of the loading equipment

The main factors influencing assembly tolerance are movements along axis $\mathrm{X}$ and the movements of adjusting attitude. Tubular-structure's movement error is influenced by rails linearly, while motions in adjusting attitude including linear movements and rotations. Motion modes and its precisions determine whether the loading equipment meet the requirements of assembly tolerances.

\subsection{Tubular-structure displacement error}

During the tubular-structure assembly the local coordinate systems have linear moves and turns relatively around the world coordinate system. The transfer matrix between the world coordinate system and the local coordinate system is as the follow.

$A^{s i}=\left[\begin{array}{cccc}\cos \psi \cos \varphi-\sin \psi \cos \theta \sin \varphi & \sin \psi \cos \varphi+\cos \psi \cos \theta \sin \varphi & \sin \theta \sin \varphi & X_{i}^{0} \\ -\cos \psi \sin \varphi-\sin \psi \cos \theta \cos \varphi & -\sin \psi \sin \varphi+\cos \psi \cos \theta \cos \varphi & \sin \theta \cos \varphi & Y_{i}^{0} \\ \sin \psi \sin \theta & -\cos \psi \sin \theta & \cos \theta & Z_{i}^{0} \\ 0 & 0 & 0 & 1\end{array}\right]$

Where $\left(\mathrm{Xi}_{0}, \mathrm{Yi}_{0}, \mathrm{Zi}_{0}\right)$ is the origin of a local coordinate system in the world coordinate system ${ }^{16}$.

When a tubular-structure is moved along the axis $\mathrm{X}$, there are three sliding move errors and three revolute errors, shown in Figure 2. The move tolerance includes a linear movement error in axial $\mathrm{X}$ direction, a linear error in axial $\mathrm{Y}$ direction, and a linear error in axial $\mathrm{Z}$ direction. The revolute errors include rolling error, pitching error, and swinging error. On the assumption that errors are of small scale, when the tubular-structure is moved along the rail, the actual position can be calculated in the following transforming matrix:

$T^{m}=\left[\begin{array}{cccc}1 & -\Delta \gamma^{m} & \Delta \beta^{m} & \delta_{x}^{m}+x_{t} \\ \Delta \gamma^{m} & 1 & -\Delta \alpha^{m} & \delta_{y}^{m} \\ -\Delta \beta^{m} & \Delta \alpha^{m} & 1 & \delta_{z}^{m} \\ 0 & 0 & 0 & 1\end{array}\right]$

\subsection{The maximum outline size of the tubu-}

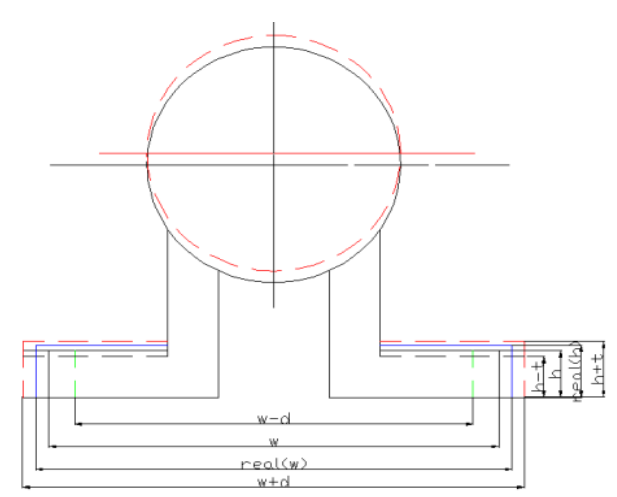

Figure 3 The outline size of tubular-structure 


\section{lar-structure}

During the loading process, the key factor influencing tubular-structure loading is the relative position between pothooks and the interior rail of the box. If the pothooks are pushed into the interior rail, and there is no interference between pothooks and the interior rail while loading, the loading is successful. Therefore, the maximum outline size of the pothook is defined as the maximum outline size of the tubular-structure. The structure of the pothook is shown in Figure 3.

During tubular-structure is pushed into the box, if the local coordinate system origin is $\left(\mathrm{x}_{0}, \mathrm{y}_{0}, \mathrm{z}_{0}\right)$, the change of $\mathrm{X}$ coordinate is defined by the linear movement distance. Because the linear movements in axis $\mathrm{Y}$ and axis $\mathrm{Z}$ are restricted by structure, the changes of coordinate are defined by the rail tolerance. Therefore, according to position transforming matrix, $\left(\mathrm{x}_{\mathrm{t}}, \mathrm{y}_{\mathrm{t}}, \mathrm{z}_{\mathrm{t}}\right)$ is calculated by the following:

$$
\left(x_{t}, y_{t}, z_{t}, 1\right)^{T}=T^{m} \bullet\left(x_{0}, y_{0}, z_{0}, 1\right)^{T}
$$

During loading, the maximum outline size of pothook is generated by the following expressions:

$$
\begin{aligned}
& W^{m}{ }_{\text {max }}=\max \left\{y_{t}\right\}-\min \left\{y_{t}\right\} \\
& H^{m}{ }_{\text {max }}=\max \left\{z_{t}\right\}-\min \left\{z_{t}\right\}
\end{aligned}
$$

According to equation (3), $\mathrm{y}_{\mathrm{t}}$ and $\mathrm{z}_{\mathrm{t}}$ are

$$
\begin{aligned}
& y_{t}=y_{0}+\Delta \gamma^{m} \bullet x_{0}-\Delta \alpha^{m} \bullet z_{0}+\delta_{y}^{m} \\
& z_{t}=z_{0}-\Delta \beta^{m} \bullet x_{0}+\Delta \alpha^{m} \bullet y_{0}+\delta_{z}^{m}
\end{aligned}
$$

The above result shows that during loading process the values of $y_{t}$ and $z_{t}$ are changed in consistent with the tolerances of rail and the support base. So, the maximum outline size of the pothook is calculated by the following equations.

$W^{m}{ }_{\text {max }}^{m}=w^{m}+\left(\Delta \gamma_{\text {max }}^{m}-\Delta \gamma_{\text {min }}^{m}\right) \bullet x_{0}+\left(\Delta \alpha_{\text {max }}^{m}-\Delta \alpha_{\text {min }}^{m}\right) \bullet z_{0}+\delta_{y \text { max }}^{m}-\delta_{y \text { min }}^{m}$ $H^{m}{ }_{\text {max }}=h^{m}+\left(\Delta \beta_{\text {max }}^{m}-\Delta \beta_{\text {min }}^{m}\right) \bullet x_{0}+\left(\Delta \alpha_{\text {max }}^{m}-\Delta \alpha_{\text {min }}^{m}\right) \bullet y_{0}+\delta_{z \max }^{m}-\delta_{z \min }^{m}$

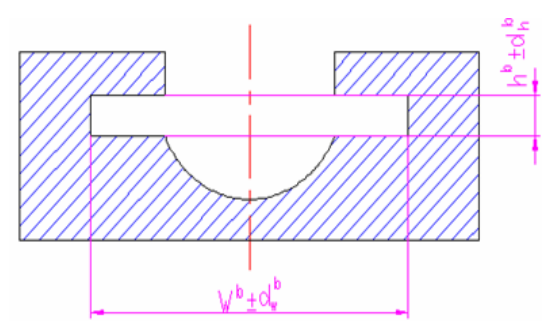

Figure 4 Cover box in section

\subsection{The minimum assembly space of the cover box}

The interior rail in section is shown in Figure 4. During loading process, the available assembly space for interior rail in cover box is decided by rail's basic size $\left(\mathrm{w}_{\mathrm{b}}, \mathrm{h}_{\mathrm{b}}\right)$, the tolerance of linear motion along axis $\mathrm{X}$, the tolerance of linear motion along axis $\mathrm{Y}$, the tolerance of linear motion along axis $\mathrm{Z}$, and the tolerance of rotating along axis $\mathrm{X}$. The minimum assembly space for the cover box is generated as

$$
\begin{aligned}
& W_{\min }^{b}=\left(w^{b}-\delta_{y \max }^{b}\right) \cdot \cos \left(\Delta \alpha^{b}\right)-\left(h^{b}-\delta_{z \max }^{b}\right) \bullet \sin \left(\Delta \alpha^{b}\right) \\
& H_{\min }^{b}=\left(h^{b}-\delta_{z \max }^{b}\right) \bullet \cos \left(\Delta \alpha^{b}\right)-\left(w^{b}-\delta_{y \max }^{b}\right) \bullet \sin \left(\Delta \alpha^{b}\right)
\end{aligned}
$$

\subsection{The tolerance model of the tubular-structure loading equipment}

The valid assembly space of the cover box is bigger than the maximum outline size of the pothook, a tubular-structure be pushed into the cover box successfully. Supposing at the beginning of tubular-structure assembly the center axis of the tubular-structure is consistent with the center axis of the box, in order to assembly tubular-structure successfully, the following conditions must be satisfied:

$$
W_{\min }^{b}>W_{\max }^{m}, H_{\min }^{b}>H_{\max }^{m}
$$

\section{Design of loading equipment}

The function of the loading equipment is to push a tubular-structure into the cover box and pull out it from the box. In order to achieve the functions and requirements, the loading equipment should have the following motions:

(i) tubular-structure moves along axis X,

(ii) cover box moves linearly along axis $\mathrm{X}$,

(iii) cover box rotates around axis $\mathrm{X}$,

(iv) cover box moves linearly along axis Z, and

(v) cover box moves linearly along axis $Y$.

Which driving system is adopted in the loading equipment? Based on assembly tolerance, the precision of driving system is defined first, the structure is configured then.

\subsection{Design of driving system in loading equipment}

Driving system of axial movement in $\mathrm{X}$ direction is composed of a motor, a retarder, a chain, and linear rails. The main factor affecting assembly quality of tubular-structure is the precision of linear rolling guides. We may figure out from equation (6) that the maximum outline size of pothook is affected by the precision of linear rolling guides. According to the precision standard of linear rolling guides JB/T7175.4-2006 ${ }^{17}$, the linear rolling guides are divided into six classes. The width of the pothook is $350 \mathrm{~mm}$, the height $20 \mathrm{~mm}$. According to the equation (6), the maximum outline sizes of the pothook are calculated under the different precision class of linear rolling guides. The calculated data are shown in Table 1. 
Table 1. The maximum outline size of pothook (mm)

\begin{tabular}{ccccccc}
\hline \multirow{2}{*}{ Size } & \multicolumn{7}{c}{ Grade } \\
\cline { 2 - 7 } & 1 & 2 & 3 & 4 & 5 & 6 \\
\hline $\begin{array}{c}\text { Maximal } \\
\text { Hight }\end{array}$ & 20.010 & 20.024 & 20.050 & 20.100 & 20.200 & 20.400 \\
\hline $\begin{array}{c}\text { Maximal } \\
\text { Width }\end{array}$ & 350.025 & 350.045 & 350.082 & 350.150 & 350.340 & 350.560 \\
\hline
\end{tabular}

In the process of assembly, there are four kinds of moves in loading cover box, which are straight move along axis $\mathrm{X}$, straight move along axis $\mathrm{Y}$, straight move along axis $\mathrm{Z}$ and rotating around axis $\mathrm{X}$. A ball screw is designed to drive cover box move straight along axis $\mathrm{X}$.. Its position tolerance is decided by the accuracy grade of the ball screw. Following national standard GB/T17587.3-1998, the accuracy grad of ball screw is divided into five classes. The valid assembly space of the cover box is not only related with the tolerance value of interior rail, but also connected with pivot position of the cover box, as shown in Figure 5. The distance between the entrance and the fore pivot is S01, S12 is the distance between the two pivots, and S20 is the distance between the back pivot and the end of the box. Due to the tolerance of ball screw in pivots, tolerances in axis $\mathrm{Y}$ at the entrance and the end of the box can be calculated according to the following equations:

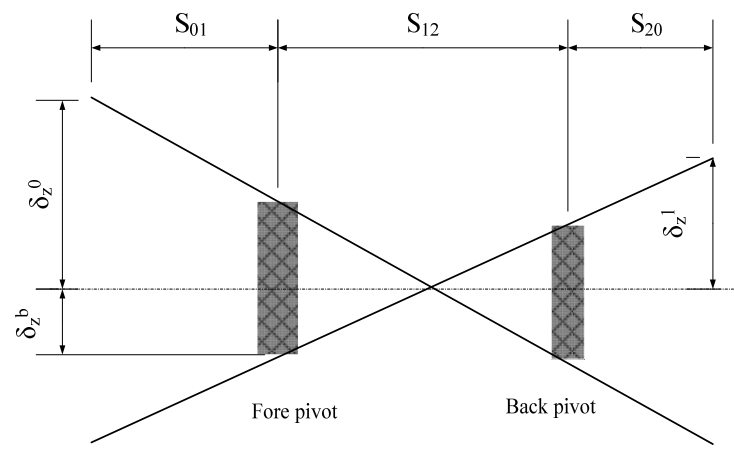

Figure 5 Tolerance analysis for the box pivots

$$
\begin{aligned}
& \delta_{Y}^{0}=\frac{2 s_{01}+s_{12}}{s_{12}} \bullet \delta_{Y}^{b} \\
& \delta_{Y}^{1}=\frac{2 s_{20}+s_{12}}{s_{12}} \bullet \delta_{Y}^{b}
\end{aligned}
$$

In the same way, the tolerance in axis $\mathrm{Z}$ at the entrance and the end of the box can be calculated as the following:

$$
\begin{aligned}
& \delta_{Z}^{0}=\frac{2 s_{01}+s_{12}}{s_{12}} \cdot \delta_{Z}^{b} \\
& \delta_{Z}^{1}=\frac{2 s_{20}+s_{12}}{s_{12}} \bullet \delta_{Z}^{b}
\end{aligned}
$$

Given S01 is 2600, S12 3400, and S20 600, the tolerance in axis $\mathrm{Y}$ under different accuracy grades of ball screws are generated as shown in Table 2, based on the equations (9) and (10).
Table 2. Tolerance in axis $\mathrm{Y}$ for the cover box $(\mu \mathrm{m})$

\begin{tabular}{ccccccc}
\hline \multirow{2}{*}{ Tolerance } & \multicolumn{7}{c}{ Grade } \\
\cline { 2 - 7 } & 1 & 2 & 3 & 4 & 5 & 6 \\
\hline The entrance & \pm 15 & \pm 20 & \pm 30 & \pm 40 & \pm 58 & \pm 15 \\
\hline The end & \pm 8 & \pm 11 & \pm 16 & \pm 22 & \pm 31 & \pm 8 \\
\hline
\end{tabular}

Data in Table 2 show the tolerance of the entrance is larger than the value of the end under the same grade of ball screw. So in the next step of analysis we only discuss the tolerance of entrance. Trapezoidal screw threads are used to move straight in axis Z. According to the national standard GB/T5796.4-2005[16], based on the equations (11) and (12), the tolerance value in axis $\mathrm{Z}$ under different accuracy grades of trapezoidal screw threads are figured out as shown in Table 3 and Table 4.

Table 3. Tolerance value in axis $\mathrm{Z}$ of box at the entrance ( $\mu \mathrm{m})$

\begin{tabular}{cccc}
\hline \multirow{2}{*}{ Pitch/mm } & \multicolumn{3}{c}{ Grade } \\
\cline { 2 - 4 } & 7 & 8 & 9 \\
\hline 3 & 898 & 1139 & 1417 \\
\hline 4 & 1012 & 1265 & 1594 \\
\hline 8 & 1341 & 1695 & 2151 \\
\hline 9 & 1417 & 1796 & 2277 \\
\hline 10 & 1417 & 1796 & 2277 \\
\hline 12 & 1594 & 2024 & 2530 \\
\hline
\end{tabular}

Table 4. Tolerance value in axis $\mathrm{Z}$ of box at the end $(\mu \mathrm{m})$

\begin{tabular}{cccc}
\hline \multirow{2}{*}{ Pitch/mm } & \multicolumn{3}{c}{ Grade } \\
\cline { 2 - 4 } & 7 & 8 & 9 \\
\hline 3 & 479 & 608 & 756 \\
\hline 4 & 540 & 675 & 851 \\
\hline 8 & 716 & 905 & 1148 \\
\hline 9 & 756 & 959 & 1215 \\
\hline 10 & 756 & 959 & 1215 \\
\hline 12 & 851 & 1080 & 1350 \\
\hline
\end{tabular}

Data in Table 3 and Table 4 show the tolerance of the entrance is larger than the value of the end under the same grade and pitch. So in the next analysis we only discuss the tolerance of the entrance. The basic size of the interior rail is $352 \mathrm{~mm} \mathrm{X} 21.5 \mathrm{~mm}$. Because of the tolerance of linear rolling guide in $\mathrm{X}$ axis, the minimum section sizes are carried out under different grades, as shown in Table 5.

Table 5. the minimum section size of interior rail in axis $\mathrm{X}(\mathrm{mm})$

\begin{tabular}{ccccccc}
\hline \multirow{2}{*}{ Size } & 1 & 2 & 3 & 4 & 5 & 6 \\
\cline { 2 - 7 } & 21.476 & 21.450 & 21.400 & 21.300 & 21.100 \\
\hline $\begin{array}{c}\text { Minimal } \\
\text { High }\end{array}$ & 21.490 & 21.00 & 351.880 & 351.700 & 351.520 \\
\hline $\begin{array}{c}\text { Minimal } \\
\text { Width }\end{array}$ & 351.994 & 351.970 & 351.940 & 350 & \\
\hline
\end{tabular}


The minimum width of the interior rail is calculated, as shown in Table 6.

Under constrain in axis $\mathrm{X}$, the available height of the interior rail is limited by the grade of trapezoidal screw threads and its pitch. When the grade is 7 , the heights of the rail under different conditions are shown in Table 7. When the grade is 8 , the heights of the rail are shown in Table 8 . When the grade is 9 , the heights of the rail are

Table 6. the minimum width of the interior rail (mm)

\begin{tabular}{ccccccc}
\hline \multirow{6}{*}{$\begin{array}{c}\text { Ball } \\
\text { screw } \\
\text { grade }\end{array}$} & 1 & 2 & 3 & 4 & 5 & 6 \\
\cline { 2 - 7 } & 351.979 & 351.955 & 351.925 & 351.865 & 351.685 & 351.505 \\
\hline 1 & 351.974 & 351.950 & 351.920 & 351.860 & 351.680 & 351.500 \\
\hline 3 & 351.964 & 351.940 & 351.910 & 351.850 & 351.670 & 351.490 \\
\hline 4 & 351.954 & 351.930 & 351.900 & 351.840 & 351.660 & 351.480 \\
\hline 5 & 351.936 & 351.912 & 351.882 & 351.822 & 351.642 & 351.462 \\
\hline
\end{tabular}

Table 7. available height of the rail when the grade is $7(\mathrm{~mm})$

\begin{tabular}{|c|c|c|c|c|c|c|}
\hline \multirow{2}{*}{ Pitch/mm } & \multicolumn{6}{|c|}{ Guide grade } \\
\hline & 1 & 2 & 3 & 4 & 5 & 6 \\
\hline 3 & 20.592 & 20.578 & 20.552 & 20.502 & 20.402 & 20.202 \\
\hline 4 & 80 & 464 & 20.438 & 20.388 & 20.288 & 20.088 \\
\hline 8 & 20.149 & 20.135 & 20.109 & 20.059 & 19.959 & 19.759 \\
\hline 9 & 20.073 & 20.059 & 20.033 & 19.983 & 19.883 & 19.683 \\
\hline 10 & 20.073 & 20.059 & 20.033 & 19.983 & 19.883 & 19.683 \\
\hline 12 & 19.896 & 19.882 & 19.856 & 19.806 & 19.706 & 19.506 \\
\hline
\end{tabular}

Table 8. available height of the rail when the grade is $8(\mathrm{~mm})$

\begin{tabular}{ccccccc}
\hline \multirow{6}{*}{ Pitch/mm } & \multicolumn{6}{c}{ Guide grade } \\
\cline { 2 - 7 } & 1 & 2 & 3 & 4 & 5 & 6 \\
\hline 3 & 20.351 & 20.337 & 20.311 & 20.261 & 20.161 & 19.961 \\
\hline 4 & 20.225 & 20.211 & 20.185 & 20.135 & 20.035 & 19.835 \\
\hline 9 & 19.795 & 19.781 & 19.755 & 19.701 & 19.601 & 19.401 \\
\hline 10 & 19.694 & 19.680 & 19.654 & 19.604 & 19.504 & 19.304 \\
\hline 12 & 19.466 & 19.452 & 19.426 & 19.376 & 19.276 & 19.076 \\
\hline
\end{tabular}


shown in Table 9.

Compared with Table 2 and Table 6, the available width of the interior rail is satisfied with the requirement of tubular-structure. Compared with Table 2 and Table 7, Table 8 and Table 9, partial data of the height are qualified, which are marked in red color.
Table 2. According to Table 6, Table 9 and equation (7), the machines for straight moving along $X$ axis, straight moving along $\mathrm{Z}$ axis and rotating around $\mathrm{X}$ axis are confirmed under the assembly tolerances. The calculated process is listed in Appendix A.

Based on the above analysis and calculation, the ma-

Table 9. Available height of rail grade 9(mm)

\begin{tabular}{cccccccc}
\hline & \multicolumn{6}{c}{ Guide grade } \\
\cline { 2 - 7 } Pitch/mm & 1 & 2 & 3 & 4 & 5 & 6 \\
\hline 3 & 20.351 & 20.337 & 20.311 & 20.261 & 20.161 & 19.961 \\
\hline 4 & 20.225 & 20.211 & 20.185 & 20.135 & 20.035 & 19.835 \\
\hline 9 & 19.795 & 19.781 & 19.755 & 19.701 & 19.601 & 19.401 \\
\hline 10 & 19.694 & 19.680 & 19.654 & 19.604 & 19.504 & 19.304 \\
\hline 12 & 19.466 & 19.452 & 19.426 & 19.376 & 19.276 & 19.076 \\
\hline
\end{tabular}

A worm and worm gear are designed to move box rotating around axis $\mathrm{X}$, which tolerance affects the available space of assembly. Assuming the distance between two axes is $160 \sim 315 \mathrm{~mm}$, according to the national standard GB/T16848-1997, if the number of threads of the worm is 2 , the number of worm gear is 50 , and the module is 8 $\mathrm{mm}$, the angle tolerance of the worm gear is obtained as shown in Table 10.

Table 10. Angle tolerance of the pitch circle (degree)

\begin{tabular}{cccc}
\hline Grade & 6 & 7 & 8 \\
\hline Tolerance & 0.024 & 0.032 & 0.041 \\
\hline
\end{tabular}

Following to the equation (7), when $\Delta \alpha^{b}$ is equal to $0.041^{\circ}$, the height is equal to $21.5 \mathrm{~mm}$, and the width is the maximum value in Table 6 , the available width is $351.447 \mathrm{~mm}$, which is larger than the maximum data in chines which are qualified to the tubular-structure assembly tolerance are listed as the following:

(i) machine for the straight line motion along axis $\mathrm{X}$ of tubular-structure is a linear rolling guide, which grade ranges from 1 to 6 ;

(ii) machine for the straight line motion along axis $\mathrm{Y}$ of the box is ball screw, which grade is from 1 to 5 ;

\subsection{The structure design for tubular-structure loading}

Based on integrated analysis of technology and economics, the mechanical structures to implement the movements are designed under constrain of the tubular-structure assembly tolerance, as shown in Figure 7. The experiment result shows that the equipment has the function of pushing a tubular-structure into the cover box under the condition of assembly tolerance.

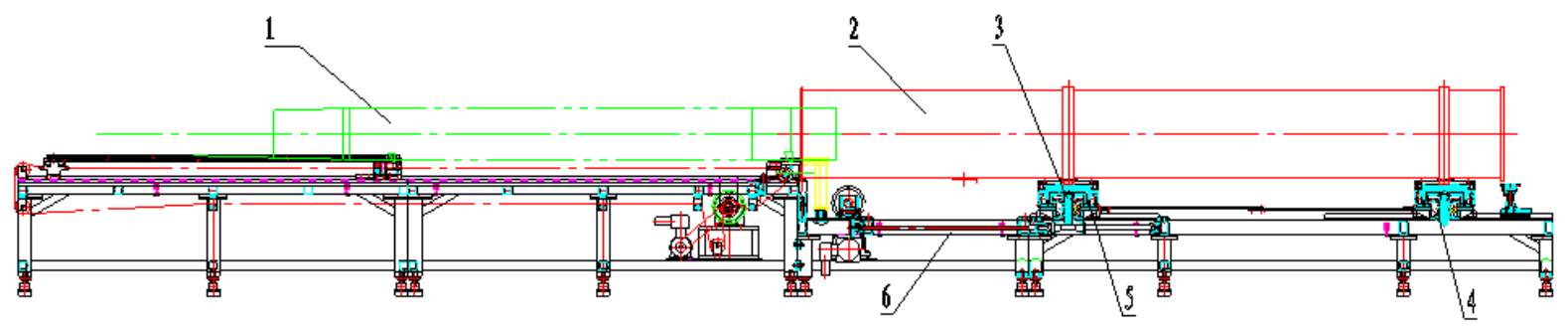

1-tubular-structure, 2-cover box, 3-trapezoidal screw thread, 4-worm and worm gear, 5-ball screw, 6-inear rolling guide Figure 7 Loading equipment of tubular-structure 


\section{Conclusions}

Based on tubular-structure assembly tolerance, this paper analyzes the assembly process of tubular-structure, and puts forward the tolerance model to design the loading equipment. The relationship between the machine tolerance of each motion and the assembly tolerance is established. Under constrain of the tubular-structure assembly tolerance, the machine and precision grade for each movement are decided. The equipment for tubular-structure loading has been designed and passed the experiment test. The tolerance model based on assembly precision for structure design is useful for other new equipment development.

\section{References}

1. Peters, J. Tolerancing the components of an assembly for minimum cost. J. Eng. Ind., 1970, 8, 677-682.

2. Speckhart, F.H. Calculation of tolerance based on a minimum cost approach. J. Eng. Ind., 1972, 5, 447-453.

3. S.S. RAO, Optimum tolerance allocation in mechanical assemblies using an interval method. Engineering Optimization Vol.37, No. 3, April 2005, 237-257.

4. Kim, S. and Knott, K. The effect of geometric tolerancing when assembling a peg into a hole using a robot. In Quality: Design, Planning and Control, presented at the Winter Annual Meeting of the ASME, Boston, MA, edited by R.E. DeVor and S.G. Kapoor, pp. 191-203, 1987 (ASME: NY).

5. Doydum, C. and Perreira, N.D. Use of Monte Carlo simulation to select dimensions, tolerances, and precision for automatic assembly. J. Manuf. Syst., 1991, 10, 209-222.

6. Cho, N. and Tu, J.F. Quantitative circularity tolerance analysis and design for 2D high precision assemblies. Int. J. Mach. Tools Manuf., 2002, 42, 1391-1401.

7. Esa Makelainen, Juhani Heilala. Assembly process level tolerance analysis for electromechanical products, Proceedings of the 4th IEEE international symposium on assembly and task planning, 2001:405-410.

8. Frederic Cazals, Jean-Claude Latombe. Effect of tolerancing on the relative positions of parts in an assembly, Proceedings of the 1997 IEEE international conference on Robotics and Automation, 1997, 606-611.

9. Mejbri H, Anselmetti B, Mawussi B. A recursive tolerancing method with sub-assembly generation, Proceedings of the IEEE International Symposium Assembly and Task Planning, 2003: 235-240.

10. Sankar Jayaram, Kevin Lyons. VADE: A virtual assembly design environment, Virtual reality. IEEE computer graphics and applications. 1999:44-50.

11. Zhao J, Masood S. Intelligent computer-aided assembly process planning system, International Journal of Advanced Manufacturing Technology, 1999, 15(5):332-337.

12. Laperriere L, EIMaraghy H A. GAPP: a generative assembly process planner, Journal of Manufacturing Systems, 1996, 15(4):282-293.

13. Tonshoff $\mathrm{H} \mathrm{K}$ et al. A knowledge-based system for automated assembly planning, Annals of the CIRP, 1992, 41(1):19-24.

14. Boothroyd G. Product design for manufacturing and assembly, Computer-Aided Design, 1994,26(7)505-520.
15. R. Sampath kumar, N. Alagumurthi. Integrated total cost and Tolerance Optimization with Generic Algorithm, International Journal of Computational Intelligence Systems, Vol.3, No.3 (September 2010), 325-333.

16. Liu Xia, Tolerance and fit and technical measurement, (Beijing: China Machine Press, 2010).

17. Wang Wenbing, Handbook of mechanical design, (Beijing: China Machine Press, 2007). 